\title{
Detection of weak magnetic fields in two HgMn stars
}

\author{
S. Hubrig ${ }^{1} \dagger$, S. P. Järvinen ${ }^{1}$, H. Korhonen ${ }^{2}$, I. Ilyin ${ }^{1}$, M. Schöller ${ }^{3}$, E. Niemczura ${ }^{4}$, \\ S. D. Chojnowski ${ }^{5}$ \\ ${ }^{1}$ Leibniz-Institut für Astrophysik Potsdam (AIP), An der Sternwarte 16, 14482 Potsdam, Germany \\ ${ }^{2}$ European Southern Observatory, Alonso de Cordova 3107, Vitacura, Santiago, Chile \\ ${ }^{3}$ European Southern Observatory, Karl-Schwarzschild-Str. 2, 85748 Garching, Germany \\ ${ }^{4}$ Astronomical Institute, University of Wroctaw, Kopernika 11, 51-622 Wroctaw, Poland \\ ${ }^{5}$ Apache Point Observatory and New Mexico State University, PO Box 59, Sunspot, NM 88340-0059, USA
}

Accepted XXX. Received YYY; in original form ZZZ

\begin{abstract}
The main-sequence mercury-manganese (HgMn) stars are known to exhibit large overabundances of exotic elements and, similar to magnetic Ap/Bp stars, are spectrum variables, implying the presence of an inhomogeneous element distribution over the stellar surface. A number of magnetic field studies have been attempted in the last decades, indicating that magnetic fields in HgMn stars, if they exist, should be rather weak. The presence of tangled magnetic fields was suggested by several authors who detected quadratic magnetic fields using the moment technique. We employ the leastsquares deconvolution technique to carry out a sensitive search for weak magnetic fields in spectropolarimetric observations of three HgMn stars, HD 221507, HD 65949, and HD 101189, which have different fundamental parameters and spectral characteristics. A definite weak longitudinal field is discovered in HD 221507 and HD 65949 on single epochs, while only marginal field detections were achieved for HD 101189. The new measurements indicate that the structure of the magnetic fields is probably rather complex: our analysis reveals the presence of reversed Stokes $V$ profiles at the same observational epoch if individual elements are used in the measurements. This is the first observational evidence that individual elements sample distinct local magnetic fields of different polarity across the stellar surface.
\end{abstract}

Key words: binaries: spectroscopic — stars: individual: HD 221507, HD 65949, HD 101189 — stars: magnetic field — stars: variables: general — stars: chemically peculiar

\section{INTRODUCTION}

The main-sequence B-type stars with spectral classes B7B9 comprise two groups of chemically peculiar stars, the classical magnetic peculiar Bp stars and the HgMn stars. The group of $\mathrm{Bp}$ stars is composed of $\mathrm{Si}$ and He-weak stars, which are usually characterised by large overabundances of Fe-peak and rare-earth elements. They possess non-axisymmetric, large-scale magnetic fields of up to several $\mathrm{kG}$ and display on their surface chemical spots of different elements. The most distinctive features of the $\mathrm{HgMn}$ group are the extreme overabundances of heavy elements, in particular of $\mathrm{Hg}$, which can reach up to 5-6 dex, and/or Mn, up to 3 dex. Other overabundance anomalies were detected for the chemical elements Y, P, Ga, Cu, Be, Bi, Zr, Sr, Xe,

† Corresponding author: shubrig@aip.de
$\mathrm{Au}$, and $\mathrm{Pt}$, whereas the elements $\mathrm{He}, \mathrm{Al}, \mathrm{Ni}$, and Co are typically underabundant (e.g., Smith 1993; Castelli \& Hubrig 2004; Hubrig et al. 2011). Notably, as of today, no theory can account satisfactorily for the observed abundance pattern in $\mathrm{HgMn}$ stars. It remains still difficult to reproduce in numerical models the detailed abundances of these stars, although some progress was achieved in the understanding of the dependence of Mn overabundances on $T_{\text {eff }}$ (e.g. Alecian \& Michaud 1981; Alecian \& Stift 2019).

Using a survey of HgMn stars in close spectroscopic binaries, Hubrig \& Mathys (1995) were the first to discuss the existence of an inhomogeneous distribution of some chemical elements over the surface of $\mathrm{HgMn}$ stars, in particular with a preferential concentration of $\mathrm{Hg}$ along the equator. Recent studies have revealed that not only $\mathrm{Hg}$, but also many other elements, most typically Ti, Cr, Fe, Mn, Sr, Y, and $\mathrm{Pt}$, are concentrated in spots of diverse sizes, and that differ- 
ent elements exhibit different abundance distributions across the stellar surface (e.g., Hubrig et al. 2006a; Briquet et al. 2010; Korhonen et al. 2013). Moreover, a few detailed studies based on extensive spectroscopic material reported the presence of dynamical abundance spot evolution of several elements at different timescales (e.g., Briquet et al. 2010; Korhonen et al. 2013; Hubrig et al. 2010).

The question of the presence of weak magnetic fields in $\mathrm{HgMn}$ stars is still under debate. It was suggested that the difficulty of spectropolarimetric studies to unambiguously detect the fields in $\mathrm{HgMn}$ stars can be attributed to the complexity of the surface magnetic field topology (Hubrig 1998a). Highly structured magnetic field topologies can reduce the Stokes $V$ signatures in spectral line profiles below the detection threshold. Furthermore, since HgMn stars usually display element spots on their surface, it is advisable to use in the magnetic field analysis line masks for individual elements separately. Combining spectral lines of various elements may lead to the dilution of the magnetic signal or even to its (partial) cancellation, if enhancements of different elements occur in regions of opposite magnetic polarity.

Attempts to detect mean longitudinal magnetic fields in HgMn stars using low- and high-resolution spectropolarimetry have been made by several authors, who reached contradictory results. While low-resolution observations do not allow the use of individual elements separately, the analysis of polarimetric spectra using the moment technique (Mathys 1993) applied to individual elements was indicative of the presence of weak longitudinal magnetic field crossover and quadratic fields (e.g., Mathys \& Hubrig 1995; Hubrig 1998a; Hubrig et al. 2012). To achieve higher accuracies in the measurements of weak magnetic fields, a line addition technique, the least-squares deconvolution (LSD; Donati et al. 1997), combining hundreds and sometimes up to thousand lines of all elements is frequently applied. The main assumption in LSD is the application of the weak field approximation, that is, the magnetic splitting of spectral lines is assumed to be smaller than their Doppler broadening. Furthermore, it is assumed that the local line profiles are self-similar and can be combined into an average profile. Due to non-linear effects in the summation and the effect of blends, the resulting LSD profiles should not be considered as observed, but rather processed Zeeman signatures. Using the lines of different elements together, Makaganiuk et al. (2011) were not able to obtain any definite detection of a mean longitudinal magnetic field in a sample of $\mathrm{HgMn}$ stars, but could decrease statistical uncertainties down to below $10 \mathrm{G}$ for the best cases. However, combining the lines of elements with different surface abundance distributions unavoidably leads to the dilution of the magnetic signal, which is expected to be rather weak in HgMn stars. Just in one case, the LSD study of the HgMn star HD 11753, did the authors use line lists for Ti, Y, and Cr separately (Makaganiuk et al. 2012). These measurements indicated a mean longitudinal magnetic field in the range from about $-20 \mathrm{G}$ to $+25 \mathrm{G}$, with measurement uncertainties of up to $25 \mathrm{G}$.

In the following sections, we present the LSD analysis of spectropolarimetric observations of three HgMn stars with different fundamental parameters and spectral characteristics, HD 221507 (= $\beta$ Scl), HD 65949, and HD 101189 (=HR 4487). HD 221507 is of special interest as a flare was discovered in the course of the inspection of Transiting Exo-
Table 1. Observation log of the three HgMn stars. The columns list the heliocentric Julian date (HJD) for the middle of the exposures and the signal-to-noise ratio $(S / N)$ of the spectra measured at about $5200 \AA$.

\begin{tabular}{crrr}
\hline \hline HJD & $S / N$ & HJD & $S / N$ \\
$2400000+$ & \multicolumn{3}{c}{$2400000+$} \\
\hline \multicolumn{3}{c}{ HD 221507 } & \multicolumn{2}{c}{ HD 65949 } \\
55210.549 & 526 & 55600.708 & 166 \\
56128.935 & 541 & 58649.479 & 147 \\
56146.791 & 186 & 58652.477 & 86 \\
56491.678 & 464 & & \\
56491.898 & 581 & HD 101189 \\
56492.667 & 578 & 54983.474 & 63 \\
56492.896 & 505 & 55201.863 & 456 \\
58646.936 & 365 & 56491.521 & 581 \\
58650.810 & 370 & 56492.481 & 599 \\
58652.931 & 406 & & \\
\hline
\end{tabular}

planet Survey Satellite (TESS) light curves by Balona et al. (2019). According to Arcos et al. (2017), HD 221507 is not a Be star, as it lacks $\mathrm{H} \alpha$ emission and has a low $v \sin i$. The fundamental parameters $T_{\text {eff }}=12400 \mathrm{~K}$ and $\log g=$ 3.90 were determined by Ghazaryan \& Alecian (2016). Furthermore, Balona et al. (2019) detected the rotation frequency for this star, $\nu_{\mathrm{rot}}=0.522 \mathrm{c} / \mathrm{d}$ using high-precision short-cadence (2 min) photometric observations assembled by TESS (Balona et al. 2019). In spite of the fact that spectral variability was already detected in numerous spectroscopical studies of $\mathrm{HgMn}$ stars, only very few $\mathrm{HgMn}$ stars have well determined rotation periods. HD 221507 was observed using the High Accuracy Radial velocity Planet Searcher polarimeter (HARPSpol; Snik et al. 2008) on ten different epochs from 2010 to 2019.

The star HD 65949 with the atmospheric parameters $T_{\text {eff }}=13100 \mathrm{~K}$ and $\log g=4.0$ (Ghazaryan \& Alecian 2016) was studied in detail by Cowley et al. (2010). It belongs to the young open cluster NGC 2516 and is a SB1 system with an orbital period of $21.2 \mathrm{~d}$. Interestingly, the authors also detected a small variation in the centre-of-mass velocity, which was interpreted as due to the presence of a third body. This makes HD 65949 a triple system. HD 65949 exhibits enormous enhancements of the elements rhenium and osmium through mercury $(\mathrm{Z}=75-80)$. According to Cowley et al. (2010), osmium and rhenium are rarely enhanced to the point where they are identified in ground-based spectra of HgMn stars. The authors suggested that the high abundance in HD 65949 must result from a very recent transfer of material. HD 65949 was observed with HARPSpol on three different epochs from 2011 to 2019.

HD 101189 with the atmospheric parameters $T_{\text {eff }}=$ $11020 \mathrm{~K}$ and $\log g=3.9$ (Ghazaryan \& Alecian 2016) is not known to belong to a SB system, but seems to be a member of a visual binary. Schöller et al. (2010) detected a close companion candidate at a separation of $0.337^{\prime \prime}$ and a position angle of $104.1^{\circ}$ using adaptive optics near-infrared observations. HD 101189 appears to be a good representative of typical $\mathrm{HgMn}$ stars, exhibiting a distinct variability of line profiles of several elements (Hubrig et al. 2011). HD 101189 


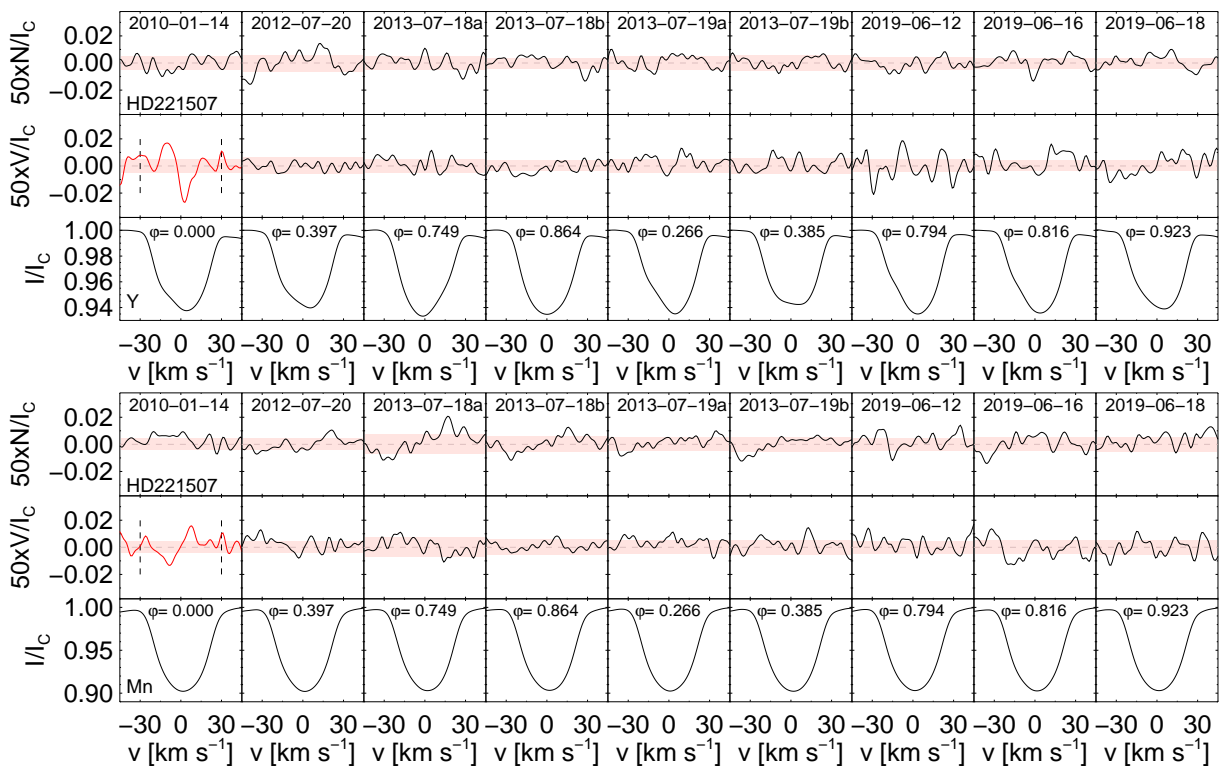

Figure 1. LSD Stokes $I$ (bottom), Stokes $V$ (middle), and diagnostic null (N) profiles (top) for HD 221507 using the Y line mask (upper panel) and the Mn line mask (bottom panel). The widths of the shaded red bands indicate the $\pm 1 \sigma$ ranges. Corresponding rotation phases for each observation are presented on top of the Stokes $I$ profiles.

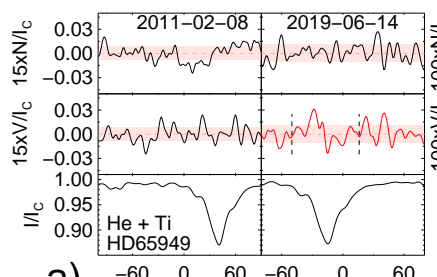

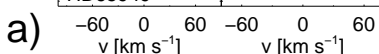

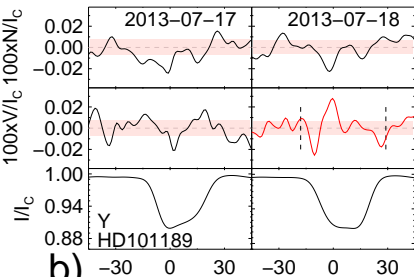

b) $-30 \underset{\mathrm{v}\left[\mathrm{km} \mathrm{s}^{-1}\right]}{0} 30 \quad-30 \underset{\mathrm{v}\left[\mathrm{km} \mathrm{s}^{-1}\right]}{0} 30$

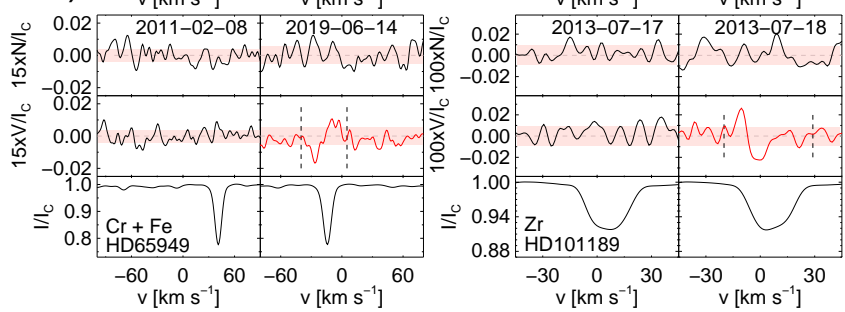

Figure 2. LSD Stokes $I$ (bottom), Stokes $V$ (middle), and diagnostic null (N) profiles (top). Left side: Results for HD 65949 using the $\mathrm{He}+\mathrm{Ti}$ line mask (upper panel) and the $\mathrm{Cr}+\mathrm{Fe}$ line mask (lower panel). Right side: Results for HD 101189 using the $\mathrm{Y}$ line mask (upper panel) and the $\mathrm{Zr}$ line mask (lower panel).

was observed with HARPSpol on four different epochs from 2009 to 2013.

\section{DATA REDUCTION AND MAGNETIC FIELD MEASUREMENT RESULTS}

HARPSpol observations of the three HgMn stars were obtained during several observing campaigns organized by different groups of observers from 2009 to 2019. Each spectropolarimetric observation usually consists of subexposures observed at different positions of the quarter-wave retarder plate. The resolving power of HARPS is about 115000 and the spectra cover the spectral range $3780-6910 \AA$, with a small gap between $5259 \AA$ and $5337 \AA$. The data reduction was performed using the HARPSpol data reduction software available on La Silla and at ESO Garching. The normalization of the spectra to the continuum level is described in detail by Hubrig et al. (2013). The heliocentric Julian date (HJD) for the middle of the exposures and the corresponding signal-to-noise ratio $(S / N)$ of the HARPS Stokes $I$ spectra measured at about $5200 \AA$ are presented in Table 1 .

Line masks for a number of elements known from several previous studies to be inhomogeneously distributed over the surface of $\mathrm{HgMn}$ stars were obtained from the Vienna Atomic Line Database (VALD; e.g., Ryabchikova et al. 2015). They were constructed for each star based on their stellar parameters given in Sect. 1. The considered line masks belong to the elements $\mathrm{He}, \mathrm{Ti}, \mathrm{Cr}, \mathrm{Fe}, \mathrm{Mn}, \mathrm{Y}$, and $\mathrm{Zr}$, which frequently show an inhomogeneous distribution on the surface of HgMn stars. The major problem in the search for very weak magnetic fields is the proper line identification of blend free spectral lines. The quality of the selection varies strongly from star to star, depending on binarity, line broadening, and the richness of the spectrum. We have checked that each line in the selected line mask is present in the stellar spectra. Obvious line blends and lines in telluric regions were excluded from the line masks. The mean longitudinal magnetic field is calculated by computing the first-order moment of the Stokes $V$ profile according to Mathys (1989).

The resulting LSD profiles for the HARPSpol spectra obtained with the best $S / N$ are shown in Figs. 1 and 2 . To classify the magnetic field detection in the LSD technique, False Alarm Probabilities (FAPs) are commonly used (Donati, Semel \& Rees 1992): a profile with FAP $<10^{-5}$ is considered a definite detection, with $10^{-5}<$ FAP $<10^{-3}$ a marginal detection, and with FAP $>10^{-3}$ a non-detection. A positive mean longitudinal magnetic field $\left\langle B_{z}\right\rangle=35 \pm 4 \mathrm{G}$ $\left(\right.$ FAP $\left.<2.8 \times 10^{-7}\right)$, is definitely detected in the spectra 

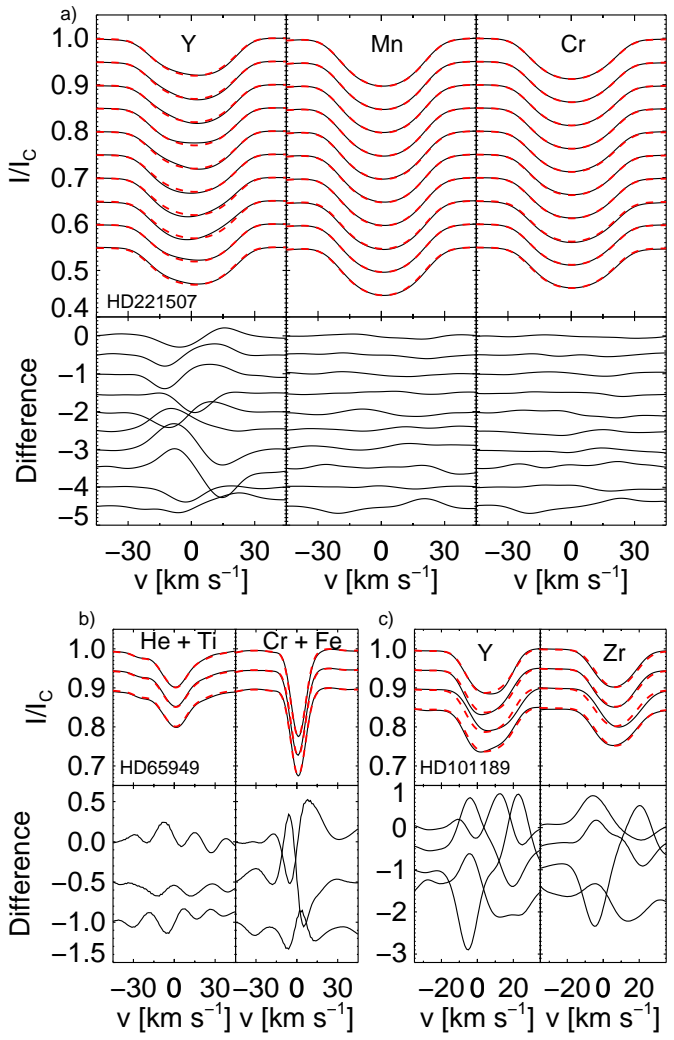

Figure 3. Variability of the LSD Stokes I profiles belonging to different elements in HD 221507 (a), HD 65949 (b), and HD 101189 (c). The profiles obtained on different nights are overplotted by the mean profiles (red dashed lines) and offset vertically for better visibility. They are presented in chronological order with the oldest observations at the bottom. The lower panels present the differences between the individual SVD Stokes $I$ profiles and the average profiles, multiplied by a factor of 100 .

of HD 221507 acquired in 2010 (HJD 2455210.549) using the $\mathrm{Y}$ line mask. Only a marginal detection of a field of negative polarity, $\left\langle B_{z}\right\rangle=-16 \pm 3 \mathrm{G}\left(\mathrm{FAP}<1.6 \times 10^{-5}\right)$ was achieved at the same epoch using the Mn line mask. Interestingly, Hubrig et al. (2012) applied the moment technique to a sample of $\mathrm{Y}$ lines observed in the HARPSpol spectra obtained at the same epoch and reported $\left\langle B_{z}\right\rangle=78 \pm 25 \mathrm{G}$, which is in good agreement with the presented LSD measurements for this element, $\left\langle B_{z}\right\rangle=35 \pm 5 \mathrm{G}$, within the error bars. The variability of the spectral lines of a few elements is presented in Fig. 3a. Obviously, the most variable lines in the spectra of this star belong to $\mathrm{Y}$.

HD 65949 exhibits the strongest longitudinal magnetic field up to $\left\langle B_{z}\right\rangle=187 \pm 36 \mathrm{G}\left(\mathrm{FAP}<7.1 \times 10^{-6}\right)$ measured using the He+Ti line mask, and up to $\left\langle B_{z}\right\rangle=-130 \pm 22 \mathrm{G}$ $\left(\mathrm{FAP}<2.3 \times 10^{-7}\right)$ using the $\mathrm{Cr}+\mathrm{Fe}$ line mask. The detection of a mean longitudinal magnetic field in this star is in agreement with the previous detection $\left(\left\langle B_{z}\right\rangle=-290 \pm 62 \mathrm{G}\right)$ by Hubrig et al. (2006b) who used the FOcal Reducer low dispersion Spectrograph (FORS 2; Appenzeller et al. 1998), installed at the ESO/VLT, in spectropolarimetric mode. The variability of the lines in both masks is displayed in Fig. 3b.

HD 101189 is $\mathrm{Y}$ and $\mathrm{Zr}$ rich and shows pronounced spectral variability (Fig. 3c). According to Hubrig et al.
(2010), also many other elements show variability of their line profiles. The analysis yields marginal detections with $\left\langle B_{z}\right\rangle=7 \pm 4 \mathrm{G}\left(\mathrm{FAP}<7.1 \times 10^{-5}\right)$ using the $\mathrm{Y}$ line mask and $\left\langle B_{z}\right\rangle=9 \pm 5 \mathrm{G}\left(\mathrm{FAP}<1.2 \times 10^{-4}\right)$ using the $\mathrm{Zr}$ line mask.

No indication of a Zeeman signature was found in any null spectrum, yielding formal non-detections with FAP > $10^{-3}$. Null spectra are usually obtained by combining the different polarizations in such a way that polarization cancels out.

\section{DISCUSSION}

The detection of a positive polarity of the longitudinal magnetic field $\left\langle B_{z}\right\rangle$ measured using the $\mathrm{Y}$ line mask and the marginal detection of a field of negative polarity measured using the Mn line mask in the first HARPSpol observation of HD 221507 acquired in 2010 (HJD 2455210.549) indicates the possible presence of local tangled magnetic fields, which are sampled differently by different elements. Similar to the results of our analysis of HD 221507, opposite field polarities are detected using different line masks for HD 65949. A number of studies using the moment technique already indicated the presence of tangled magnetic fields in a few HgMn stars. However, due to the weakness of the longitudinal magnetic fields in these stars, the detections of the presence of complex fields was exclusively based on the discovery of quadratic magnetic fields (e.g., Mathys \& Hubrig 1995; Hubrig 1998a; Hubrig et al. 2012). The presence of opposite field polarities in temperature spots in the late-type star II Peg was recently discussed by Strassmeier, Carroll \& Ilyin (2019) and appears to be typical in magnetically active latetype stars. However, the detection of opposite field polarities using line masks for different elements is unexpected. Although it is tempting to assume that the detection of a flare in HD 221507 by Balona et al. (2019) is related to the presence of a complex magnetic field structure similar to that typically observed in active low-mass stars, there is a possibility that the observed flare originated in the closeby late-type companion at a separation of $0.641^{\prime \prime}$ detected by Schöller et al. (2010). On the other hand, previous observations of $13 \mathrm{HgMn}$ stars, including HD 221507, with the ROSAT High-Resolution Imager confirmed X-ray sources at the position of these stars (Hubrig 1998b). Future high spatial resolution observations (e.g. with Chandra) are needed to determine whether the origin of the observed X-ray emission is related to the HgMn stars themselves or to late-type companions.

Since we have not discovered magnetic fields in all the observations obtained at different epochs distributed over several years, it is quite possible that their detectability strongly depends on the spot position and the element abundance within the spot, i.e. we probably observe different "evolutionary states" of the spots, especially when observing epochs are separated by long time intervals.

In our analysis we detect that Zeeman features in the Stokes $V$ profiles of HD 65949 and HD 101189 appear shifted bluewards. This probably indicates that the observed polarization signal corresponds to a fraction of the visible hemisphere and could be related to a surface inhomogeneous distribution of the individual chemical elements. Similar blue- 
ward shifts of Zeeman features persisting for more than half a rotation period were also observed in the magnetic Bp star 36 Lyn showing surface element patches (Wade et al. 2006). If the measured magnetic fields are associated with an inhomogeneous distribution of the elements on the stellar surface, then the field strengths measured in the longitudinal strips present just a lower limit for the field strength expected to be measured in the chemical spots directly.

The possibility that the detected Zeeman features originate in close-by late-type companions to these stars can be discarded as the visual close companion to HD 101189 was never detected spectroscopically. According to the orbital radial velocities of HD 65949 presented by Cowley et al. (2010), the observed Zeeman feature cannot be related to the secondary of the SB1 system. The spectrum can still be contaminated by the distant third component, but apart from the center-of-mass velocity changes there is no trace of this component in the available spectra.

Possible scenarios for the generation of magnetic fields in HgMn stars, which are frequently in binaries and multiple systems, were already discussed in the past by Hubrig et al. (2011). While classical magnetic Bp stars in close binaries are very rare, more than $2 / 3$ of the $\mathrm{HgMn}$ stars are known to belong to spectroscopic binaries, with a preference of orbital periods ranging from 3 to 20 days (Hubrig \& Mathys 1995). Surveying a magnitude limited sample of late B-type stars using the ninth catalogue of spectroscopic binary orbits by Pourbaix et al. (2004) reveals only very few normal late-B type primaries in systems with orbital periods below $20 \mathrm{~d}$ (Hubrig \& Mathys 1995). Based on these statistics, it cannot be excluded that most late B-type stars formed in binary systems with certain orbital parameters become HgMn stars (Hubrig et al. 2011). Hubrig et al. (2011) suggested that a tidal torque varying with depth and latitude in a star induces differential rotation. Differential rotation in radiative chemically peculiar stars can be prone to magnetorotational instability (MRI). Magnetohydrodynamical simulations by Arlt, Hollerbach, \& Rüdiger (2003) revealed a distinct structure for the magnetic field topology similar to the fractured elemental rings observed on the surface of HgMn stars. The introduction of a magnetic field excites the MRI on a very short time-scale when compared to the timescale of microscopic magnetic diffusion. Although the fields are not strong, complex surface patterns can be obtained from the nonlinear, nonaxisymmetric evolution of the MRI.

Due to the weakness of the detected magnetic fields, future observing campaigns should be based on high-resolution spectropolarimetric observations obtained at a very high $S / N$ and have to be carried out during rather short time intervals of the order of a few weeks: As has already been shown by Briquet et al. (2010) and Korhonen et al. (2013), chemical spots on the surface of $\mathrm{HgMn}$ stars are not stable and show temporal evolution of the spot shape and the element abundance on time scales as short as a few months. Briquet et al. (2010) found that Y, Sr, and Ti spots change their configuration on HD 11753 between two data sets that were separated by approximately two months, thus providing clear evidence of dynamical spot evolution on a short timescale. Korhonen et al. (2013) investigated further the reliability of that fast dynamical spot evolution reported for HD 11753, and confirmed, both from chemical spot maps and from equivalent width measurements, that the spot configuration changed on a timescale of months.

\section{ACKNOWLEDGEMENTS}

We would like to dedicate this work to our late colleague Dr. Fiorella Castelli. We thank the referee for the insightful questions and suggestions. Based on observations made with ESO Telescopes at the La Silla Paranal Observatory under programme IDs 083.D-1000, 084.D-0338, 086.D-0240, 187.D0917, 089.D-0383, 091.D-0759, and 0103.C-0240. This work has made use of the VALD database, operated at Uppsala University, the Institute of Astronomy RAS in Moscow, and the University of Vienna, and of the SIMBAD database, operated at CDS, Strasbourg, France.

\section{REFERENCES}

Alecian G., Michaud G., 1981, ApJ, 245, 226

Alecian G., Stift M. J., 2019, MNRAS, 482, 4519

Appenzeller I., et al., 1998, The ESO Messenger, 94, 1

Arcos C., Jones C. E., Sigut T. A. A., Kanaan S., Curé M., 2017, ApJ, 842, 48

Arlt R., Hollerbach R., Rüdiger G., 2003, A\&A, 401, 1087

Balona L. A., et al., 2019, MNRAS, 485, 3457

Briquet M., Korhonen H., González J. F., Hubrig S., Hackman T., 2010, A\&A, 511, A71

Castelli F., Hubrig S., 2004, A\&A, 425, 263

Cowley C. R., Hubrig S., Palmeri P., Quinet P., Biémont É., Wahlgren G. M., Schütz O., González J. F., 2010, MNRAS, 405, 1271

Donati J.-F., Semel M., Rees D. E., 1992, A\&A, 265, 669

Donati J.-F., Semel M., Carter B. D., Rees D. E., Collier Cameron A., 1997, MNRAS, 291, 658

Ghazaryan S., Alecian G., 2016, MNRAS, 460, 1912

Hubrig S., Mathys G., 1995, Comm. on Astroph., 18, 167

Hubrig S., 1998a, Contr. of the Astron. Obs. Skal. Pleso, 27, 296

Hubrig S., Berghöfer T. W., Mathys, G., 1998b, Contr. of the Astron. Obs. Skalnate Pleso, 27, 464

Hubrig S., González J. F., Savanov I., Schöller M., Ageorges N., Cowley C. R., Wolff B., 2006a, MNRAS, 371, 1953

Hubrig S., North P., Schöller M., Mathys G., 2006b, Astron. Nachr., 327, 289

Hubrig S., et al., 2010, MNRAS, 408, L61

Hubrig S., et al., 2011, Astron. Nachr., 332, 998

Hubrig S., et al., 2012, A\&A, 547, A90

Hubrig S., Ilyin I., Schöller M., Lo Curto G., 2013, Astr. Nachr., 334, 1093

Korhonen H., et al., 2013, A\&A, 553, A27

Makaganiuk V., et al., 2011, A\&A, 525, A97

Makaganiuk V., et al., 2012, A\&A, 539, A142

Mathys G., 1989, Fundam. Cosm. Phys., 13, 143

Mathys G., 1993, Astron. Soc. of the Pacific, 44, 232

Mathys G., Hubrig S., 1995, A\&A, 293, 810

Pourbaix D., et al., 2004, A\&A, 424, 727

Ryabchikova T., Piskunov N., Kurucz R. L., Stempels H. C., Heiter U., Pakhomov Y., Barklem P. S., 2015, Phys. Scr., 90, 054005

Schöller M., Correia S., Hubrig S., Ageorges N., 2010, A\&A, 522, A85

Smith K. C., 1993, A\&A, 276, 393

Snik F., Jeffers S., Keller C., Piskunov N., Kochukhov O., Valenti J., Johns-Krull C., 2008, SPIE Conf. Series, 7014, 701400

Strassmeier K. G., Carroll T. A., Ilyin I. V., 2019, A\&A, 625, A27

Wade G. A., et al. 2006, A\&A, 458, 569 


\section{L6 Hubrig et al.}

This paper has been typeset from a $\mathrm{T}_{\mathrm{E}} \mathrm{X} / \mathrm{LAT}_{\mathrm{E}} \mathrm{X}$ file prepared by the author. 